\title{
Relationship between aspirin use of esophageal, gastric and colorectal cancer patient survival: a meta-analysis
}

Ju-Li Lin ${ }^{1,2+}$, Jian-Xian Lin ${ }^{1,2,3 \dagger}$, Chao-Hui Zheng ${ }^{1,2}$, Ping Li ${ }^{1,2,3}$, Jian-Wei Xie ${ }^{1,2}$, Jia-bin Wang ${ }^{1,2,3}$, Jun Lu,2, Qi-Yue Chen ${ }^{1,2}$, Long-long Cao ${ }^{1,2}$, Mi Lin ${ }^{1,2}$ and Chang-Ming Huang ${ }^{1,2,3^{*}}$ (D)

\begin{abstract}
Background: Many studies have found that use of aspirin can lengthen survival in patients with gastrointestinal cancer. The aim of this study was to assess the survival benefit of aspirin use compared with non-aspirin use for patients with esophageal, gastric or colorectal cancer.

Methods: We searched online databases, including PubMed, the Cochrane Library, Embase and wnw.clinicaltrials.gov for studies that were conducted, before April 30th, 2020, to identify relevant studies. Overall sunvival and cancer-specific survival of esophageal, gastric and colorectal cancers among aspirin users were compared with those among non-aspirin users. Data extraction and quality evaluation were independently conducted by 2 investigators. A meta-analysis was performed to calculate the pooled risk ratios (RRs) for overall sunvival and cancer-specific survival by using either a fixed-effects model or a random-effects model.

Results: A total of 18 studies were included in this meta-analysis, with more than 74,936 patients. There were no significant differences between postdiagnosis aspirin use and overall survival for esophageal and gastric cancers. For colorectal cancer, a benefit that was associated with postdiagnosis aspirin use was observed for overall survival and cancer-specific survival $[\mathrm{HR}=0.83,95 \% \mathrm{Cl}(0.75,0.9) ; \mathrm{HR}=0.78,95 \% \mathrm{Cl}(0.66,0.92)$, respectively. However, a prediagnosis of aspirin use did not provide a benefit for overall or cancer-specific survival in colorectal cancer. HR values for overall and cancer-specific survival benefits for colorectal cancer associated with both prediagnosis and postdiagnosis aspirin were as follows: $\mathrm{HR}=0.75,95 \% \mathrm{Cl}(0.61,0.92)$ and $\mathrm{HR}=0.78,95 \% \mathrm{CI}(0.73,0.85)$, respectively. In addition, the survival benefit of postdiagnosis aspirin use appeared to be confined to patients with mutated PIK3CA tumors [HR $=0.78,95 \% \mathrm{Cl}(0.50$, 0.99)] and was positive for PTGS2 (COX-2) expression [HR $=0.75,95 \% \mathrm{Cl}(0.43,1.30)]$.

Conclusions: These findings provide further indications that postdiagnosis aspirin use improves overall survival and cancer-specific survival in colorectal cancer, especially for patients who are positive for PTGS2 (COX-2) expression and PIK3CA-mutated tumors. However, aspirin therapy does not improve overall survival in esophageal and gastric cancers, although the meta-analysis was mainly limited to retrospective studies.
\end{abstract}

Keywords: Aspirin, Esophageal cancer, Gastric cancer, Colorectal cancer, Long-term survival

\footnotetext{
* Correspondence: hcmlr2002@163.com

${ }^{\dagger}$ Ju-Li Lin and Jian-Xian Lin contributed equally to this work and should be considered co-first authors.

'Department of Gastric Surgery, Fujian Medical University Union Hospital, No.29 Xinquan Road, Fuzhou 350001, Fujian Province, China

${ }^{2}$ Department of General Surgery, Fujian Medical University Union Hospital, Fuzhou, Fujian Province, China

Full list of author information is available at the end of the article
}

(c) The Author(s). 2020 Open Access This article is licensed under a Creative Commons Attribution 4.0 International License, which permits use, sharing, adaptation, distribution and reproduction in any medium or format, as long as you give appropriate credit to the original author(s) and the source, provide a link to the Creative Commons licence, and indicate if changes were made. The images or other third party material in this article are included in the article's Creative Commons licence, unless indicated otherwise in a credit line to the material. If material is not included in the article's Creative Commons licence and your intended use is not permitted by statutory regulation or exceeds the permitted use, you will need to obtain permission directly from the copyright holder. To view a copy of this licence, visit http://creativecommons.org/licenses/by/4.0/ The Creative Commons Public Domain Dedication waiver (http://creativecommons.org/publicdomain/zero/1.0/) applies to the data made available in this article, unless otherwise stated in a credit line to the data. 


\section{Background}

Esophageal, gastric and colorectal cancers are the most common cancers of the digestive tract [1]. Many factors, including old age and poor living habits, are risk factors for gastrointestinal malignancies. Although the incidence and mortality of gastrointestinal malignancies have been reduced in recent years, the comprehensive treatment of gastrointestinal malignancies has progressed slowly in recent decades. Therefore, it is urgent to discover a more effective comprehensive treatment for gastrointestinal malignancies. Aspirin is a nonselective cyclooxygenase inhibitor with strong antipyretic and analgesic effects and is widely used for its anti-inflammatory and anti-rheumatic properties. For example, small doses of aspirin are used to prevent the onset of cardiovascular disease, cerebrovascular disease and transient ischemic attacks. In recent years, many studies [2-7] have found that aspirin also has anticancer effects. However, as there are still some controversy about these studies, the aim of this study was to assess the survival benefits of aspirin use (compared with non-aspirin use) for esophageal, gastric and colorectal cancer patients through the use of a meta-analysis.

\section{Methods}

All of the search results were evaluated according to the Preferred Reporting Items for Systematic Reviews and Meta-Analyses (PRISMA 2009) statement [8].

\section{Inclusion and exclusion criteria}

The inclusion criteria were as follows: (1) RCTs or observational studies including cohort and case-control studies; (2) the outcomes of interest beingdefined as OS (overall survival) and CSS (cancer-specific survival) of esophageal, gastric, colorectal, colon or rectal cancer; (3) the study addressing aspirin usage at the times of prediagnosis and/or postdiagnosis of esophageal, gastric, colorectal, colon or rectal cancer; (4) HR or OR estimates with 95\% CIs were available. The exclusion criteria were as follows: (1) duplicate articles; (2) inadequate data; and (3) sample sizes less than 20; (4) NOS $\leq 5$.

\section{Literature search}

We conducted a comprehensive systematic literature search of online databases, including PubMed, the Cochrane Library, Embase and www.clinicaltrials.gov for studies that were conducted before April 30th, 2020, to identify all RCTs and observational studies. The following key words were used in these literature searches: ('colorectal cancer' or 'colon cancer' or 'rectal cancer' or 'colorectal adenocarcinoma' or 'colon adenocarcinoma' or 'rectal adenocarcinoma') AND ('aspirin' or 'non-steroidal anti-inflammatory drugs' or 'NSAIDS') ('gastric cancer' or 'gastric adenocarcinoma') AND ('aspirin' or 'non-steroidal anti-inflammatory drugs' or 'NSAIDS') ('esophageal cancer' or 'esophageal adenocarcinoma' or 'esophageal squamous cell carcinoma') AND ('aspirin' or 'non-steroidal anti-inflammatory drugs' or 'NSAIDS'). There were no language restrictions. We also reviewed the references of the included articles and of the related systematic reviews, in order to identify additional studies.

\section{Study selection and quality assessment}

The qualities of the included non-RCTs were assessed by using the Newcastle-Ottawa Scale (NOS) [9]. The scale utilizes a score system ranging from 0 to 9 , and the quality of the observational studies were considered to be high-quality with a score of 5 or higher.

\section{Data extraction}

Data extraction and the evaluation of the quality of the literature were independently conducted by 2 investigators (Ju-li Lin and Jian-xian Lin). At time when there was any uncertainty about the inclusion of a study, the issue was discussed between the two investigators to achieve a resolution. A Microsoft Excel database was employed to record all of the available information, including the baseline details, title, first author's name, year of publication, study design, region, journal, sample size, period of patient recruitment, follow-up time, and HRs.

\section{Statistical analysis}

The Cochran's Q statistic and $\mathrm{I}^{2}$ statistics were applied to assess the heterogeneity among all of the studies [10]. For the $\mathrm{Q}$ statistic, a $p$ value of less than 0.1 was considered to be statistically significant. When statistical heterogeneity was detected, the sources of the heterogeneity were explored, and sensitivity analyses were performed. A random-effects model was used if heterogeneity existed; otherwise, the fixed-effect model was used. When possible, subgroup analyses were conducted to assess the potential impacts of the mutation statuses. The cut-off point for quality among observational studies (NOS $\leq 5$ vs. NOS $>5$ ) was arbitrarily defined. Publication bias was assessed using the Begg and Egger regression asymmetry test, together with funnel plots. All of the statistical analyses were conducted by using STATA, version 13.0 (Stata Corporation, College Station, TX).

\section{Results}

\section{Retrieved studies and characteristics}

According to the previously described search strategy, 3612 citations were obtained from the online database up until April 30th, 2020. A total of 3569 articles were excluded by viewing the titles and abstracts. The full texts of 36 records were read. Ultimately, 18 full-text studies [4-7, 11-24] were obtained and assessed according to the eligibility criteria, including 1 case-control 
study and 17 cohort studies, with the studies comprised of more than 74,936 patients. The detailed literature search and screening process are shown in Supplement Figure 1. The characteristics included in the study are shown in Tables 1 and 2, including the first author's name, year of publication, study design, region, journal, sample size, period of patient recruitment patients, follow-up time and definition of aspirin use.

The qualities of 18 studies was assessed by using NOS; four studies achieved a score of 6 , six studies achieved a score of 7 and eight studies achieved a score of 8 (Tables 3 and 4). Thirteen studies stated a clear follow-up time. The longest median follow-up period was 10.8 years. Six studies reported a clear definition of the use of PPIs. Seven studies compared the risk of gastric cancer between PPI users and non-PPI users. Thirteen studies evaluated the association between prediagnosis aspirin use and colorectal cancer survival. Thirteen studies evaluated the association between postdiagnosis aspirin use and colorectal cancer survival.

\section{Association between postdiagnosis aspirin use and survival (OS and CSS) in esophageal and gastric cancers}

Three studies (involving 6797 patients) compared the overall survival of esophageal cancer among aspirin users compared with non-aspirin users. The estimated pooled HRs showed no significant differences between the two groups $[\mathrm{HR}=1.009,95 \% \mathrm{CI}(0.847,1.202)]$ (Fig. 1a).

Two studies (involving 4589 patients) compared the overall survival of gastric cancer among aspirin users compared with non-aspirin users, and the estimated pooled HRs indicated no significant differences between the groups $[H R=0.870,95 \% \mathrm{CI}(0.470,1.610)]$ (Fig. 1a).

Three studies (involving 11,380 patients) compared the overall survival of upper digestive cancer among aspirin users compared with non-aspirin users, with no significant differences between the two groups based on estimated pooled HRs $[\mathrm{HR}=0.831,95 \% \mathrm{CI}(0.679,1.016)]$ (Fig. 1a).

One study (involving 946 patients) compared the cancer-specific survival of esophageal cancer among aspirin users with non-aspirin users; based on HRs, the use of aspirin postdiagnosis was associated with longer cancer-specific survival $[\mathrm{HR}=0.34,95 \% \mathrm{CI}(014,0.69)]$ (Fig. 1b). One study involving 750 patients compared the cancer-specific survival of gastric cancer among aspirin users with non-aspirin users, and the HRs revealed no significant differences between the groups $[H R=0.70$, 95\% CI $(0.29,1.69)]$ (Fig. 1b).

\section{Association between postdiagnosis aspirin use and survival (OS and CSS) in colorectal cancer}

Ten studies (involving 67,552 patients) compared the overall survival of colorectal cancer among aspirin users compared with non-aspirin users. According to the estimated pooled HRs, the use of aspirin postdiagnosis was associated with longer overall survival $[H R=0.83$, $95 \% \operatorname{CI}(0.75,0.93)]$ (Fig. 2a).

The result of cumulative meta-analysis showed that the significant difference supporting PPI use was first found in the latest study in Joseph et al. $[\mathrm{HR}=0.89,95 \%$ $\mathrm{CI}(0.86-0.93)]$, with the CI narrowing and the effect size becoming stable (Fig. $2 \mathrm{~b}$ ).

Eight studies (involving 52,662 patients) compared cancer-specific survival in colorectal cancer among aspirin users and non-aspirin users. The estimated pooled HRs showed that the use of aspirin postdiagnosis was associated with longer overall survival $[\mathrm{HR}=0.78$, $95 \% \mathrm{CI}(0.66,0.92)]$ (Fig. 2c).

The result of cumulative meta-analysis indicated that the significant difference supporting PPI use was first found in the latest study by Joseph et al. [HR $=0.85,95 \%$ CI (0.80-0.89], with the CI narrowing and the effect size becoming stable (Fig. 2d).

\section{Association between prediagnosis aspirin use and survival (OS and CSS) in colorectal cancer}

With regard to overall survival in colorectal cancer, five studies involving 6202 patients compared among aspirin users compared with non-aspirin users. The estimated pooled HRs demonstrated no significant differences between the two groups $[\mathrm{HR}=1.01,95 \% \mathrm{CI}(0.96,1.06)]$ (Fig. 3a).

Five studies (involving 45,101 patients) compared the cancer-specific survival of colorectal cancer among aspirin users compared with non-aspirin users, and according to the estimated pooled HRs, there were no significant differences between the groups $[\mathrm{HR}=0.93$, $95 \% \mathrm{CI}(0.84,1.03)]$ (Fig. 3b).

\section{Association between both prediagnosis and postdiagnosis aspirin use and survival (OS and CSS) in colorectal cancer}

Four studies (involving 2350 patients) compared the overall survival of colorectal cancer among aspirin users compared with non-aspirin users. The estimated pooled HRs revealed that the use of aspirin both prediagnosis and postdiagnosis was associated with longer overall survival $[\mathrm{HR}=0.75,95 \% \mathrm{CI}(0.61,0.92)]$ (Fig. 4a).

Three studies (involving 1849 patients) compared cancer-specific survival in colorectal cancer among aspirin users compared with non-aspirin users, and the estimated pooled HRs indicated that the use of aspirin both prediagnosis and postdiagnosis was associated with longer overall survival $[\mathrm{HR}=0.78,95 \% \mathrm{CI}(0.73,0.85)]$ (Fig. 4b).

\section{Subgroup analysis according to the PIK3CA gene status} Four studies (involving 4346 patients) compared the overall survival of colorectal cancer among aspirin users compared with non-aspirin users among those with 


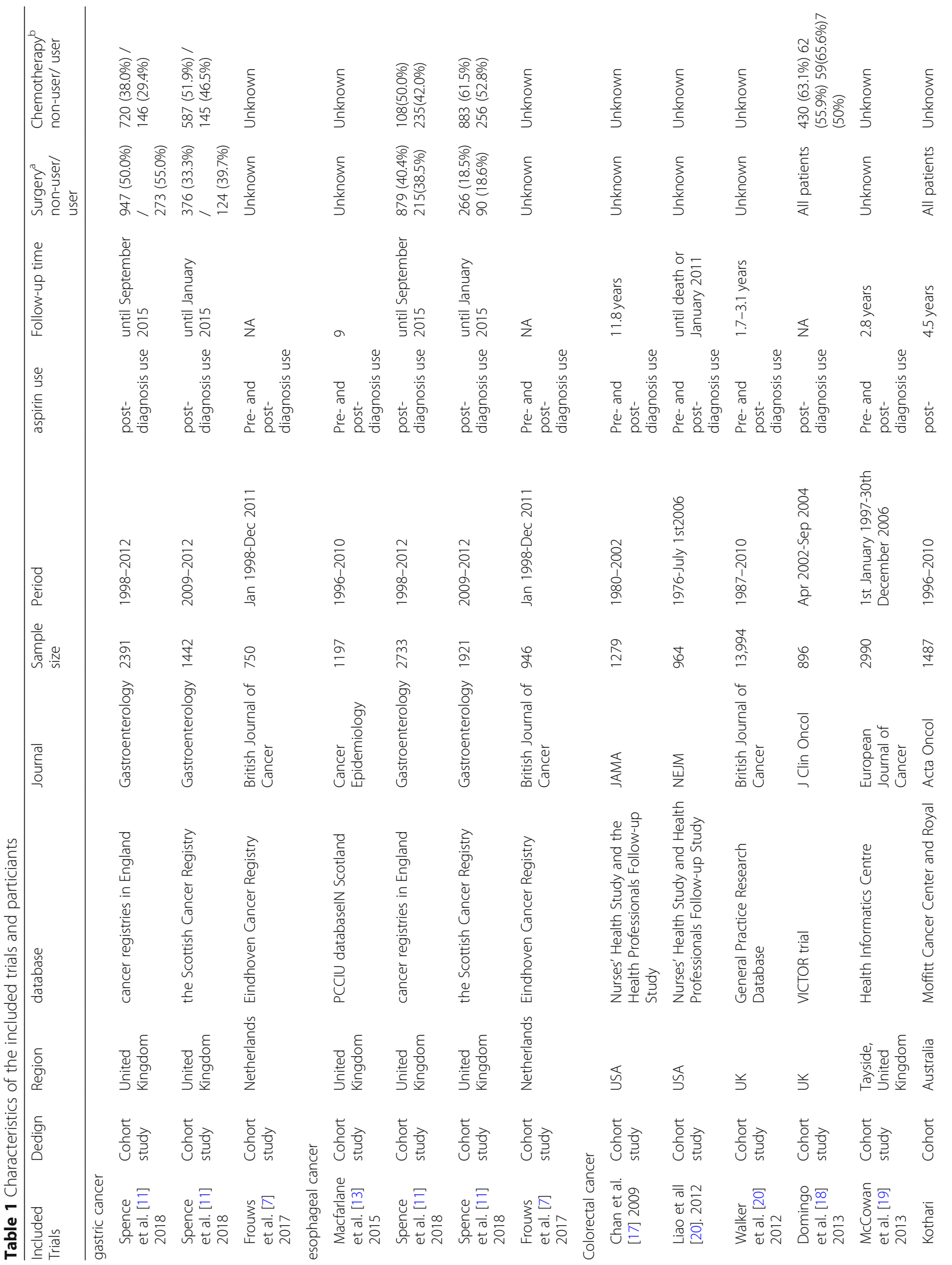




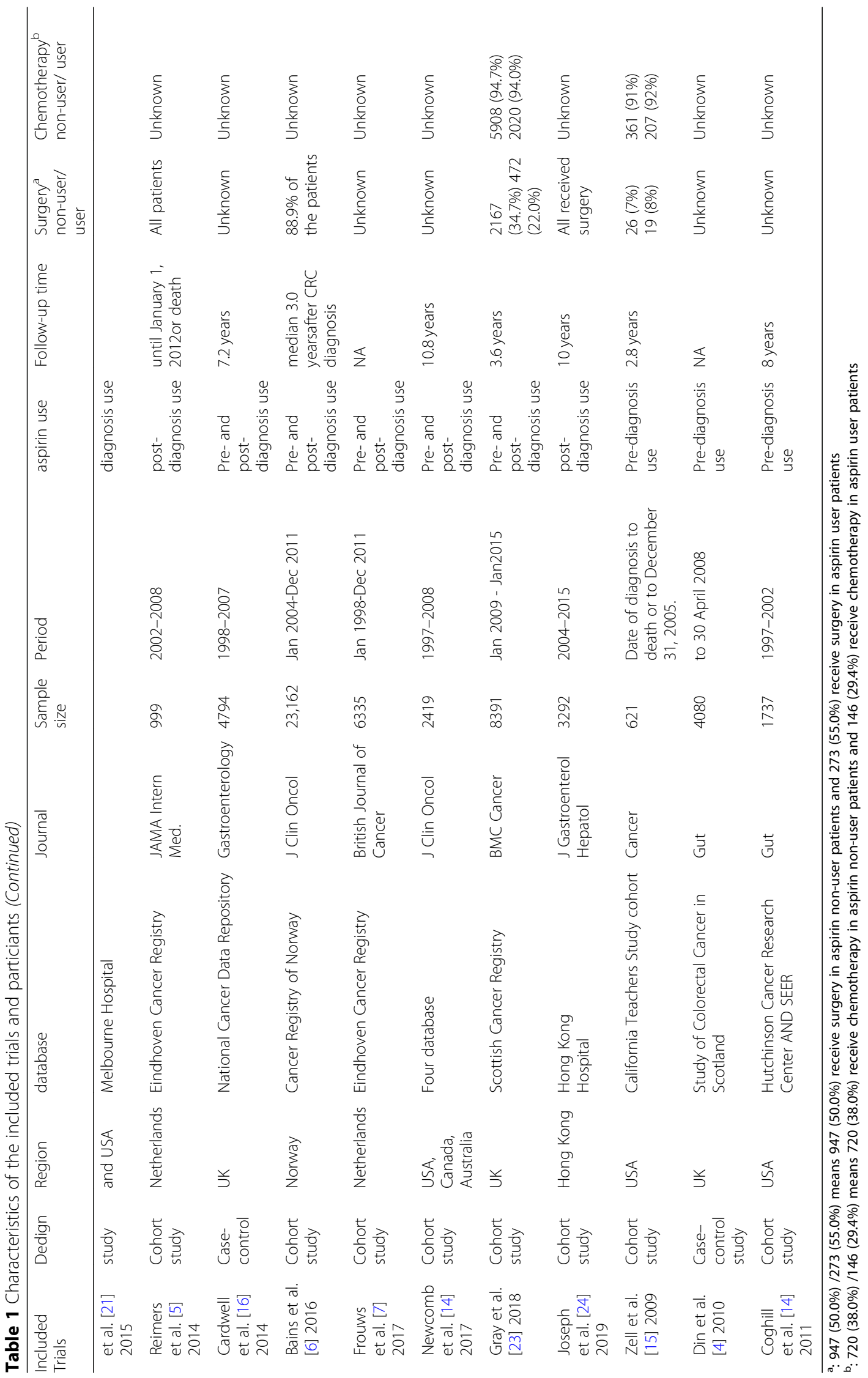


Table 2 Characteristics of the included trials and particiants

\begin{tabular}{|c|c|c|c|c|c|}
\hline $\begin{array}{l}\text { Included } \\
\text { Trials }\end{array}$ & $\begin{array}{l}\text { Stage }^{a} \\
\text { non-user/ } \\
\text { user }\end{array}$ & Dosage & Duration & Reason & Outcomes \\
\hline \multicolumn{6}{|c|}{ gastric cancer } \\
\hline $\begin{array}{l}\text { Spence } \\
\text { et al. [11] } \\
2018\end{array}$ & $\begin{array}{l}\text { I } 28(1.5 \%) \\
12(2.4 \%) \\
\text { || } 43(2.3 \%) \\
20(4.0 \%) \\
\text { III } 59(3.1 \%) \\
16(3.2 \%) \\
\text { IV } 119(6.3 \%) \\
16(3.2 \%) \\
\text { Missing } 1646 \\
\text { (86.9\%) } \\
432(87.1 \%)\end{array}$ & Low-dose aspirin (75 mg) use & $\begin{array}{l}182,365,548 \\
\text { and } 730 \text { tablets }\end{array}$ & Unknown & $\begin{array}{l}\text { not associated with increased } \\
\text { survival in sophageal or gastric } \\
\text { cancer }\end{array}$ \\
\hline $\begin{array}{l}\text { Spence } \\
\text { et al. [11] } \\
2018\end{array}$ & Unknown & Low-dose aspirin (75 mg) use & $\begin{array}{l}182,365,548 \\
\text { and } 730 \text { tablets }\end{array}$ & Unknown & $\begin{array}{l}\text { not associated with increased } \\
\text { survival in sophageal or gastric } \\
\text { cancer }\end{array}$ \\
\hline $\begin{array}{l}\text { Frouws } \\
\text { et al. [7] } \\
2017\end{array}$ & Unknown & $\begin{array}{l}\text { Nonusers were defined as } \\
\text { patients who received for less } \\
\text { than } 30 \text { days or never used } \\
\text { aspirin. }\end{array}$ & Unknown & Unknown & $\begin{array}{l}\text { increased survival } \\
\text { in cancers }\end{array}$ \\
\hline \multicolumn{6}{|c|}{ esophageal cancer } \\
\hline $\begin{array}{l}\text { Macfarlane } \\
\text { et al. [13] } \\
2015\end{array}$ & Unknown & Unknown & Unknown & Unknown & improved survival was observed \\
\hline $\begin{array}{l}\text { Spence } \\
\text { et al. [11] } \\
2018\end{array}$ & $\begin{array}{l}\text { I } 34(1.6 \%) \\
10(1.8 \%) \\
\text { II } 69(3.2 \%) \\
28(5.0 \%) \\
\text { III } 183(8.4 \%) \\
47(8.4 \%) \\
\text { IV } 132(6.1 \%) \\
23(4.1 \%) \\
\text { Unknown } \\
1756(80.8 \%) \\
451(80.7 \%)\end{array}$ & Low-dose aspirin (75 mg) use & $\begin{array}{l}182,365,548 \\
\text { and } 730 \text { tablets }\end{array}$ & Unknown & $\begin{array}{l}\text { not associated with increased } \\
\text { survival in sophageal or gastric } \\
\text { cancer }\end{array}$ \\
\hline $\begin{array}{l}\text { Spence } \\
\text { et al. [11] } \\
2018\end{array}$ & Unknown & Low-dose aspirin (75 mg) use & $\begin{array}{l}182,365,548 \\
\text { and } 730 \text { tablets }\end{array}$ & Unknown & $\begin{array}{l}\text { not associated with increased } \\
\text { survival in sophageal or gastric } \\
\text { cancer }\end{array}$ \\
\hline $\begin{array}{l}\text { Frouws } \\
\text { et al. [7] } \\
2017\end{array}$ & Unknown & $\begin{array}{l}\text { Nonusers were defined as } \\
\text { patients who received for less } \\
\text { than } 30 \text { days or never used } \\
\text { aspirin. }\end{array}$ & Unknown & Unknown & increased survival in cancers \\
\hline \multicolumn{6}{|c|}{ Colorectal cancer } \\
\hline $\begin{array}{l}\text { Chan et al. } \\
\text { [17] } 2009\end{array}$ & $\begin{array}{l}\text { | } 228(32 \%) \\
193(35 \%) \\
\text { || } 260(36 \%) \\
186(33 \%) \\
\text { I|I } 231(32 \%) \\
181(32 \%) \\
\text { | } 218(30 \%) \\
203(37 \%) \\
\text { ||265(36\%) } \\
181(33 \%) \\
\text { I|I } 247(34 \%) \\
165(30 \%)\end{array}$ & $\begin{array}{l}\text { used aspirin } 2 \text { or more timesper } \\
\text { week }\end{array}$ & Unknown & $\begin{array}{l}\text { Headache, arthritis } \\
\text { and other } \\
\text { musculoskeletal } \\
\text { pain, cardiovascular } \\
\text { disease }\end{array}$ & $\begin{array}{l}\text { associated with lower risk of } \\
\text { colorectal cancer-specific and overall } \\
\text { mortality }\end{array}$ \\
\hline $\begin{array}{l}\text { Liao et all } \\
\text { [20]. } 2012\end{array}$ & $\begin{array}{l}\text { I } 112(24 \%) \\
102(30 \%) \\
\text { || } 159(34 \%) \\
87(26 \%) \\
\text { III } 128(27 \%) \\
99(29 \%)\end{array}$ & $\begin{array}{l}\text { as regular use of aspirin } \\
\text { duringmost weeks }\end{array}$ & Unknown & $\begin{array}{l}\text { Headache, arthritis } \\
\text { and other } \\
\text { musculoskeletal } \\
\text { pain, cardiovascular } \\
\text { disease }\end{array}$ & $\begin{array}{l}\text { associated with longer survival } \\
\text { among patients with mutated- } \\
\text { PIK3CA colorectal cancer }\end{array}$ \\
\hline
\end{tabular}


Table 2 Characteristics of the included trials and particiants (Continued)

\begin{tabular}{|c|c|c|c|c|c|}
\hline $\begin{array}{l}\text { Included } \\
\text { Trials }\end{array}$ & $\begin{array}{l}\text { Stage }^{a} \\
\text { non-user/ } \\
\text { user }\end{array}$ & Dosage & Duration & Reason & Outcomes \\
\hline & $\begin{array}{l}\text { IV } 31(7 \%) 18 \\
(5 \%) \\
\text { Unknown } 36 \\
(8 \%) 31(9 \%) \\
\text { I } 19(20 \%) 27 \\
(41 \%) \\
\text { II } 36(38 \%) \\
19(29 \%) \\
\text { III } 23(24 \%) \\
14(21 \%) \\
\text { IV } 12(13 \%) 3 \\
(5 \%) \\
\text { Unknown } 5 \\
\text { (5\%) } 3(5 \%)\end{array}$ & & & & \\
\hline $\begin{array}{l}\text { Walker } \\
\text { et al. [20] } \\
2012\end{array}$ & Unknown & $\begin{array}{l}\text { a repeat prescription }(>2) \\
\text { within the period }\end{array}$ & $\begin{array}{l}\text { a fixed period of } 1 \text { year } \\
\text { post-diagnosis }\end{array}$ & Unknown & $\begin{array}{l}\text { have a potential as anti-neoplastics } \\
\text { in } \\
\text { diagnosed colorectal cancer }\end{array}$ \\
\hline $\begin{array}{l}\text { Domingo } \\
\text { et al. [18] } \\
2013\end{array}$ & $\begin{array}{l}\text { II } 332 \\
(48.7 \%) 57 \\
(51.4 \%) \\
\text { III } 349 \\
(51.2 \%)(54 \\
48.6 \%) \\
\text { I| } 46(51.1 \%) \\
8(57.1 \%) \\
\text { III } 44(48.9 \%) \\
6(42.9 \%)\end{array}$ & $\begin{array}{l}\text { taking regularlow-dose aspirin at } \\
\text { random assignment or who } \\
\text { started during follow-up }\end{array}$ & Unknown & $\begin{array}{l}\text { adjuvant setting of } \\
\text { colorectal cancer: }\end{array}$ & $\begin{array}{l}\text { support the prospective evaluation } \\
\text { of adjuvant low-dose aspirin in } \\
\text { patients with tumor PIK3CA mutation }\end{array}$ \\
\hline $\begin{array}{l}\text { McCowan } \\
\text { et al. [19] } \\
2013\end{array}$ & Unknown & $\begin{array}{l}28 \text { tablets at one per day gave } \\
\text { coverage for that prescription of } \\
28 \text { days. }\end{array}$ & $\begin{array}{l}\text { date of the first } \\
\text { prescription post- } \\
\text { diagnosis to the end of } \\
\text { coverage of the last } \\
\text { prescription }\end{array}$ & Unknown & $\begin{array}{l}\text { use post-diagnosis of colorectal can- } \\
\text { cer may reduce both all cause and } \\
\text { colorectal cancer specific mortality }\end{array}$ \\
\hline $\begin{array}{l}\text { Kothari } \\
\text { et al. [21] } \\
2015\end{array}$ & $\begin{array}{l}\text { | 6(4\%) 2(4\%) } \\
\text { || 50(37\%) } \\
16(33 \%) \\
\text { ||| 45(33\%) } \\
22(45 \%) \\
\text { IV 35(26\%) } \\
9(18 \%)\end{array}$ & $\begin{array}{l}\text { at least } 75 \mathrm{mg} \text { of aspirin daily at } \\
\text { the time of CRC diagnosis }\end{array}$ & Unknown & Unknown & $\begin{array}{l}\text { significant improvements in survival } \\
\text { in PIK3CA-mutated CRC patients }\end{array}$ \\
\hline $\begin{array}{l}\text { Reimers } \\
\text { et al. [5] } \\
2014\end{array}$ & $\begin{array}{l}\text { I } 95(13.8 \%) \\
38(21.2 \%) \\
\text { II } 218 \\
(31.9 \%) \\
69(38.5 \%) \\
\text { III } 219 \\
(32.0 \%) \\
57(31.8 \%) \\
\text { IV149(21.8\%) } \\
15(0.8 \%) \\
\text { Unknown } 3 \\
\text { (0.4\%) }\end{array}$ & $\begin{array}{l}\text { given a prescription for aspirin } \\
\text { for } 14 \text { days or more after a } \\
\text { colon cancerdiagnosis }\end{array}$ & Unknown & Unknown & $\begin{array}{l}\text { Increased PTGS2 expression or the } \\
\text { presence of mutated PIK3CA did not } \\
\text { predict benefit from aspirin }\end{array}$ \\
\hline $\begin{array}{l}\text { Cardwell } \\
\text { et al. [16] } \\
2014\end{array}$ & $\begin{array}{l}\text { I } 65(4.2 \%) \\
\text { II } 283 \\
(18.2 \%) \\
\text { III } 565 \\
\text { (36.2\%) } \\
\text { IV } 187 \\
(12.0 \%) \\
\text { Missing } 459 \\
(29.4 \%)\end{array}$ & $\begin{array}{l}\text { low dose if } 75 \mathrm{mg}(0.3 \% \text { of } \\
\text { prescriptions after cancer } \\
\text { diagnosis were } 25 \mathrm{mg}, 98.5 \% \\
\text { were } 75 \mathrm{mg} \text {, and } 1.2 \% \text { were } 300 \\
\mathrm{mg}) .\end{array}$ & $\begin{array}{l}\text { Duration of use was } \\
\text { determined from } \\
\text { quantity of tablets. }\end{array}$ & Unknown & $\begin{array}{l}\text { low-dose aspirin usage after } \\
\text { diagnosis of colorectal cancer did } \\
\text { not increase survival time. }\end{array}$ \\
\hline $\begin{array}{l}\text { Bains et al. } \\
\text { [6] } 2016\end{array}$ & $\begin{array}{l}\text { | } 3600 \\
(21.9 \%) 1631\end{array}$ & $\begin{array}{l}\text { three or more prescriptions of } \\
\text { aspirin starting from } 30 \text { days }\end{array}$ & $\begin{array}{l}\text { Aspirin prescriptions } \\
\text { lasted } 3 \text { months at a }\end{array}$ & Unknown & $\begin{array}{l}\text { Aspirin use after the diagnosis of } \\
\text { CRC is independently associated }\end{array}$ \\
\hline
\end{tabular}


Table 2 Characteristics of the included trials and particiants (Continued)

\begin{tabular}{|c|c|c|c|c|c|}
\hline $\begin{array}{l}\text { Included } \\
\text { Trials }\end{array}$ & $\begin{array}{l}\text { Stage }^{a} \\
\text { non-user/ } \\
\text { user }\end{array}$ & Dosage & Duration & Reason & Outcomes \\
\hline & $\begin{array}{l}(27.7 \%) \\
\text { II } 4840 \\
(29.4 \%) 2112 \\
(35.9 \%) \\
\text { III } 4829 \\
(29.3 \%) 1581 \\
(26.8 \%) \\
\text { IV } 3188 \\
(19.4 \%) 565 \\
(9.6 \%)\end{array}$ & after the diagnosis of CRC & $\begin{array}{l}\text { time (100-tablet packets, } \\
\text { one tablet once per day), }\end{array}$ & & with improved CSS and OS. \\
\hline $\begin{array}{l}\text { Frouws } \\
\text { et al. [7] } \\
2017\end{array}$ & Unknown & $\begin{array}{l}\text { Nonusers were defined as } \\
\text { patients who received for less } \\
\text { than } 30 \text { days or never used } \\
\text { aspirin. }\end{array}$ & Unknown & Unknown & increased survival in cancers \\
\hline $\begin{array}{l}\text { Newcomb } \\
\text { et al. [14] } \\
2017\end{array}$ & $\begin{array}{l}\text { I } 326(30 \%) \\
311(36 \%) \\
\text { || } 391(36 \%) \\
259(30 \%) \\
\text { III } 263(24 \%) \\
225(26 \%) \\
\text { IV } 106(10 \%) \\
617(\%) \\
\text { Unknown } \\
311,166\end{array}$ & $\begin{array}{l}\text { using the medications at least } \\
\text { twice per week for more than } 1 \\
\text { month }\end{array}$ & $\begin{array}{l}\text { Pre-diagnostic use } 1 \text { year } \\
\text { before diagnosis /post- } \\
\text { diagnostic use between } \\
\text { baseline } \\
\text { and the 5-year follow-up } \\
\text { interview }\end{array}$ & Unknown & $\begin{array}{l}\text { regular use of NSAIDs after CRC } \\
\text { diagnosis was significantly associated } \\
\text { with improved survival in individuals } \\
\text { with KRAS wild-type tumors }\end{array}$ \\
\hline $\begin{array}{l}\text { Gray et al. } \\
\text { [23] } 2018\end{array}$ & $\begin{array}{l}\text { A } \\
1683(27.0 \%) \\
597(27.8 \%) \\
\text { B } \\
2340(37.5 \%) \\
851(39.6 \%) \\
C \\
2218(35.5 \%) \\
702(32.7 \%)\end{array}$ & $\begin{array}{l}\text { Low-dose }(75 \mathrm{mg} \text { ) aspirin } \\
\text { exposure was identified from } \\
\text { dispensing } \\
\text { records within this database }\end{array}$ & $\begin{array}{l}\text { users after a lag of } 6 \\
\text { months after their first } \\
\text { aspirin } \\
\text { prescription }\end{array}$ & Unknown & $\begin{array}{l}\text { either before or after diagnosis, did } \\
\text { not prolong survival in this } \\
\text { population-based CRC cohort. }\end{array}$ \\
\hline $\begin{array}{l}\text { Joseph } \\
\text { et al. [24] } \\
2019\end{array}$ & Unknown & no less than $80 \mathrm{mg}$ per day & at least a month & Unknown & $\begin{array}{l}\text { lowers risk of both CRC-related mor- } \\
\text { tality and overall mortality }\end{array}$ \\
\hline $\begin{array}{l}\text { Zell et al. } \\
\text { [15] } 2009\end{array}$ & Unknown & $\begin{array}{l}\text { taken aspirin regularly at least } \\
\text { once a week }\end{array}$ & $\begin{array}{l}\text { the total duration of use } \\
\text { in number of years }(<1 \text {, } \\
1,2,3-4,5-9 \text {, or } 10) \text {. }\end{array}$ & Unknown & $\begin{array}{l}\text { NSAIDs are associated with } \\
\text { decreased mortality among female } \\
\text { CRC patients }\end{array}$ \\
\hline $\begin{array}{l}\text { Din et al. } \\
\text { [4] } 2010\end{array}$ & Unknown & reported intake of aspirin & Unknown & Unknown & $\begin{array}{l}\text { NSAID use prior to CRC } \\
\text { diagnosis does not influence survival } \\
\text { of colorectal cancer }\end{array}$ \\
\hline $\begin{array}{l}\text { Coghill } \\
\text { et al. [14] } \\
2011\end{array}$ & Unknown & $\begin{array}{l}\text { at least twice per week for } 1 \\
\text { month }\end{array}$ & $\begin{array}{l}\text { first, } 0-6 \text { months; second, } \\
6 \text { monthse } 2.5 \text { years; third, } \\
2.5-7 \text { years; fourth, }>7 \\
\text { years). }\end{array}$ & Unknown & $\begin{array}{l}\text { regular use of } \\
\text { NSAIDs prior to diagnosis is } \\
\text { associated with improved } \\
\text { colorectal cancer survival }\end{array}$ \\
\hline
\end{tabular}

a: stage I $28(1.5 \%) 12(2.4 \%)$ means $28(1.5 \%)$ are stage I aspirin non-user patients and $12(2.4 \%)$ are stage I aspirin user patients

PIK3CA gene mutation. Based on the estimated pooled HRs, the use of aspirin postdiagnosis was associated with longer overall survival $[\mathrm{HR}=0.70,95 \% \mathrm{CI}(0.50,0.99)]$ (Fig. 5a).

For overall survival in colorectal cancer, three studies involving 8490 patients compared among aspirin users compared with non-aspirin users among patients with a wild-type PIK3CA gene, and the estimated pooled HRs showed no significant differences between the groups $[\mathrm{HR}=0.79,95 \% \mathrm{CI}(0.53,1.13)]$ (Fig. 5a).

Two studies involving 2451 patients compared the cancer-specific survival in colorectal cancer among aspirin users compared with non-aspirin users among patients with a mutated PIK3CA gene. The estimated pooled HRs showed that the use of aspirin postdiagnosis was associated with longer overall survival $[\mathrm{HR}=0.27$, 95\% CI $(0.08,0.91)]$ (Fig. 5b).

\section{Subgroup analysis according to the PTGS2 (COX-2) expression status}

Two studies involving 560 patients compared overall survival in colorectal cancer among aspirin users compared with non-aspirin users in patients with strong 
Table 3 Quality assessment of the observational studies using the Newcastle-Ottawa Scale (NOS). Assessment of the cohort studies

\begin{tabular}{|c|c|c|c|c|c|c|c|c|c|c|c|}
\hline Author & year & $\begin{array}{l}\text { Representativeness } \\
\text { of the exposed } \\
\text { cohort }\end{array}$ & $\begin{array}{l}\text { Selection } \\
\text { of the } \\
\text { non- } \\
\text { exposed } \\
\text { cohort }\end{array}$ & $\begin{array}{l}\text { Ascertainment } \\
\text { of exposure to } \\
\text { implants }\end{array}$ & $\begin{array}{l}\text { Demonstration } \\
\text { that outcome } \\
\text { of interest was } \\
\text { not present at } \\
\text { start of study }\end{array}$ & $\begin{array}{l}\text { Comp } \\
\text { of cor }\end{array}$ & $\begin{array}{l}\text { rability } \\
\text { prts }\end{array}$ & $\begin{array}{l}\text { Assessment } \\
\text { of outcome }\end{array}$ & $\begin{array}{l}\text { Was follow } \\
\text { up long } \\
\text { enough for } \\
\text { outcomes } \\
\text { to occur }\end{array}$ & $\begin{array}{l}\text { Adequacy } \\
\text { of follow } \\
\text { up of } \\
\text { cohorts }\end{array}$ & $\begin{array}{l}\text { Total } \\
\text { score }\end{array}$ \\
\hline $\begin{array}{l}\text { Chan et al. } \\
\text { [17] }\end{array}$ & 2009 & + & + & + & + & + & - & + & + & + & 8 \\
\hline $\begin{array}{l}\text { Coghill } \\
\text { et al. [14] }\end{array}$ & 2011 & + & + & + & + & + & - & + & + & + & 8 \\
\hline $\begin{array}{l}\text { Bains et al. } \\
\text { [6] }\end{array}$ & 2016 & + & + & + & + & + & - & + & + & + & 8 \\
\hline $\begin{array}{l}\text { Liao et all } \\
\text { [20]. }\end{array}$ & 2012 & + & + & + & + & + & - & + & + & + & 8 \\
\hline $\begin{array}{l}\text { Walker } \\
\text { et al. [20] }\end{array}$ & 2012 & + & + & - & - & + & + & + & - & + & 6 \\
\hline $\begin{array}{l}\text { Domingo } \\
\text { et al. [18] }\end{array}$ & 2013 & + & + & - & + & + & + & + & - & + & 7 \\
\hline $\begin{array}{l}\text { Frouws } \\
\text { et al. [7] }\end{array}$ & 2017 & + & + & - & + & + & + & + & - & + & 7 \\
\hline $\begin{array}{l}\text { Gray et al. } \\
\text { [23] }\end{array}$ & 2018 & + & + & - & - & + & - & + & + & + & 6 \\
\hline $\begin{array}{l}\text { Kothari } \\
\text { et al. [21] }\end{array}$ & 2014 & + & + & - & - & + & - & + & + & + & 6 \\
\hline $\begin{array}{l}\text { McCowan } \\
\text { et al. [19] }\end{array}$ & 2013 & + & + & - & + & + & + & + & - & + & 7 \\
\hline $\begin{array}{l}\text { Macfarlane } \\
\text { et al. [13] }\end{array}$ & 2015 & + & + & - & - & + & - & + & + & + & 6 \\
\hline $\begin{array}{l}\text { Newcomb } \\
\text { et al. [14] }\end{array}$ & 2017 & + & + & + & + & + & - & + & + & + & 8 \\
\hline $\begin{array}{l}\text { Reimers } \\
\text { et al. [5] }\end{array}$ & 2014 & + & + & - & + & + & + & + & - & + & 7 \\
\hline $\begin{array}{l}\text { Spence } \\
\text { et al. [11] }\end{array}$ & 2017 & + & + & + & + & + & + & + & - & + & 8 \\
\hline $\begin{array}{l}\text { Zell et al. } \\
\text { [15] }\end{array}$ & 2009 & + & + & - & + & + & + & + & - & + & 7 \\
\hline $\begin{array}{l}\text { Joseph } \\
\text { et al. [24] }\end{array}$ & 2019 & + & + & - & + & + & + & + & - & + & 7 \\
\hline
\end{tabular}

PTGS2 (COX-2) expression. According to the estimated pooled HRs, the use of aspirin postdiagnosis was associated with longer overall survival $[\mathrm{HR}=0.65,95 \% \mathrm{CI}(0.54$, 0.83)] (Fig. 5c).

Regarding the overall survival of colorectal cancer, two studies involving 4328 patients compared aspirin users with non-aspirin users among patients with weak PTGS2
(COX-2) expression. The estimated pooled HRs showed no significant differences between the two groups $[\mathrm{HR}=$ 0.75, 95\% CI(0.43, 1.30)] (Fig. 5c).

\section{Subgroup analysis according tumor stage}

Four studies involving 28,032 patients compared overall survival in colorectal cancer among aspirin users

Table 4 Quality assessment of the observational studies using the Newcastle-Ottawa Scale (NOS). Assessment of the case-control study

\begin{tabular}{|c|c|c|c|c|c|c|c|c|c|c|}
\hline Author & year & $\begin{array}{l}\text { Is the case } \\
\text { definition } \\
\text { adequate }\end{array}$ & $\begin{array}{l}\text { Representativeness } \\
\text { of the cases }\end{array}$ & $\begin{array}{l}\text { Selection } \\
\text { of } \\
\text { Controls }\end{array}$ & $\begin{array}{l}\text { Definition } \\
\text { of } \\
\text { Controls } \\
\end{array}$ & Comparability & $\begin{array}{l}\text { Ascertainment } \\
\text { of exposure }\end{array}$ & $\begin{array}{l}\text { Same method of } \\
\text { ascertainment for } \\
\text { cases and controls }\end{array}$ & $\begin{array}{l}\text { Non- } \\
\text { Response } \\
\text { Rate } \\
\end{array}$ & $\begin{array}{l}\text { Total } \\
\text { score }\end{array}$ \\
\hline $\begin{array}{l}\text { Cardwell } \\
\text { et al. [16] }\end{array}$ & 2014 & + & + & + & + & + & + & + & + & 8 \\
\hline $\begin{array}{l}\text { Din et al. } \\
{[4]}\end{array}$ & 2010 & + & + & + & + & + & + & + & + & 8 \\
\hline
\end{tabular}




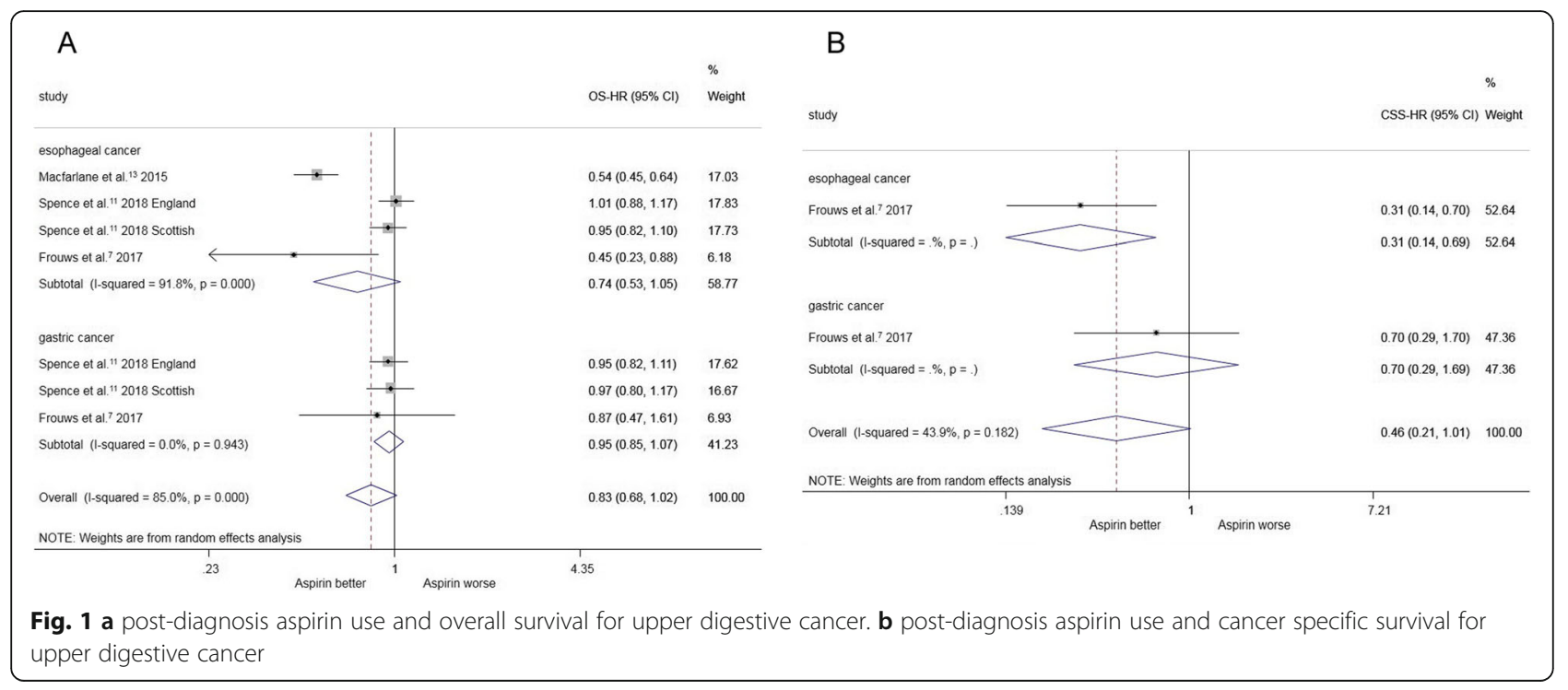

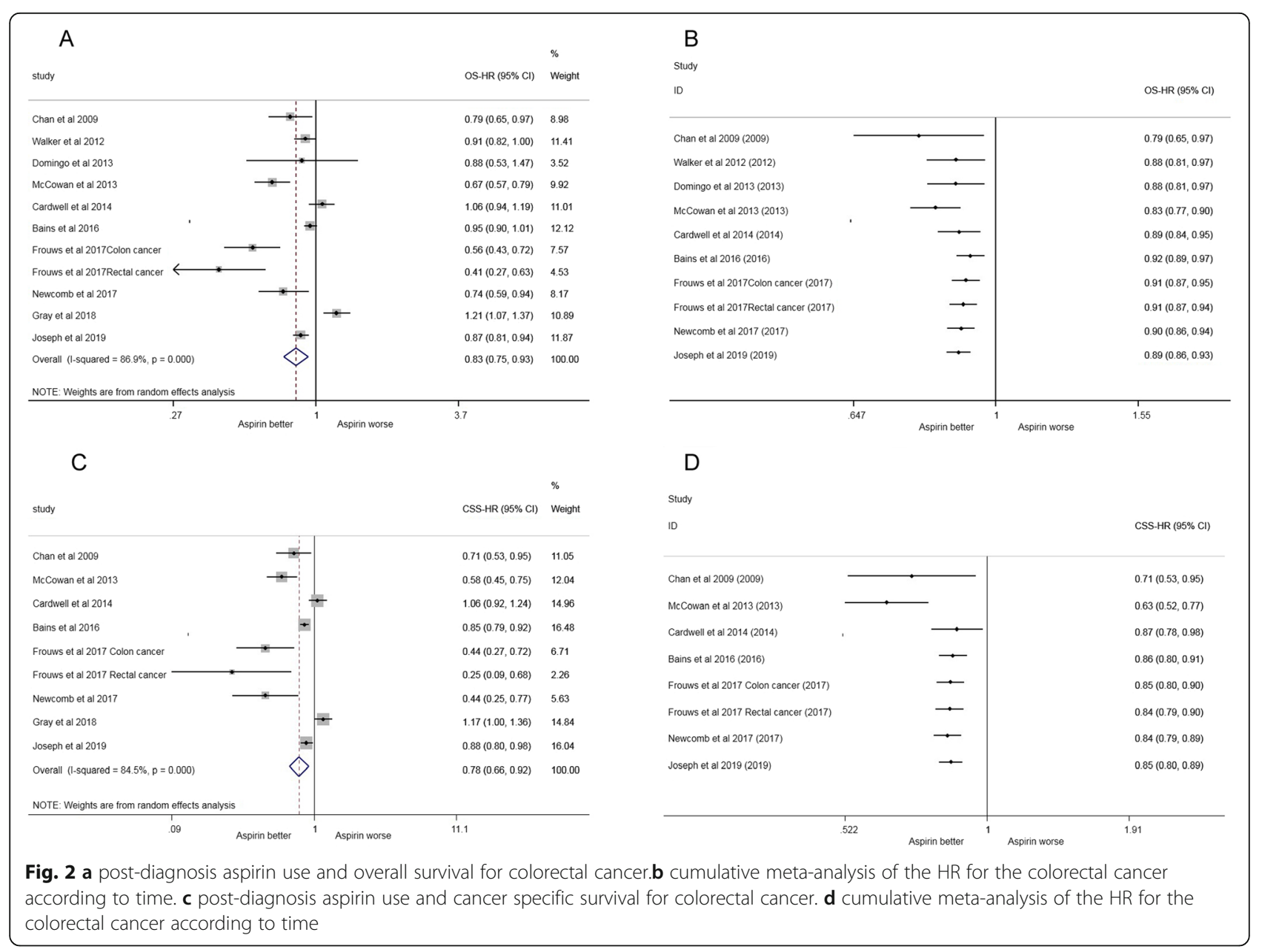




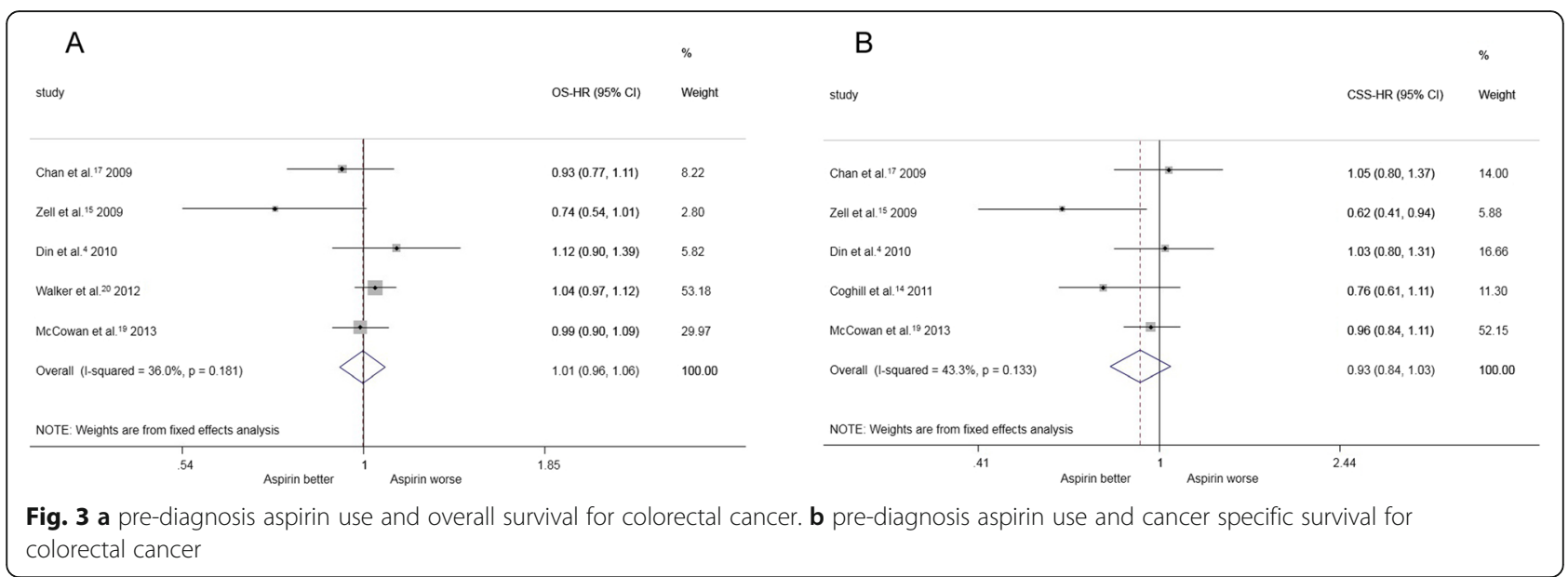

compared with non-aspirin users among patients. The estimated pooled HRs showed no significant differences between the groups (Supplement Figure 3A).

Five studies involving 32,826 patients compared cancer specific survival in colorectal cancer among aspirin users compared with non-aspirin users. The estimated pooled HRs showed no significant differences between the groups in stage I, stage III and stage IV patients. While the use of aspirin was associated with longer cancer specific survival in stage II patients $[\mathrm{HR}=0.65,95 \% \mathrm{CI}(0.54$, 0.83)] (Supplement Figure 3B).

\section{Sensitivity analysis}

Sensitivity analysis was performed to test the stability of the results by excluding each study successively. The results were not affected by sequential exclusion of any particular trial, except for one study (Bains et al., 2016). The detailed sensitivity analysis results are depicted in Fig. 6 .

\section{Publication bias}

In a meta-analysis with few studies (less than 10), the power of asymmetrical tests is too low to distinguish chance from real asymmetry. Because of the limited number of included studies, it was difficult to confirm the existence of publication bias in the current metaanalysis.

\section{Discussion}

Aspirin is a nonselective cyclooxygenase inhibitor. Many studies [2-7] have observed that aspirin can improve the prognosis of digestive malignant tumors. However, there were some controversial issues in these studies, especially among those studies that focused on esophageal, gastric, and colorectal cancers with different gene mutation types, such as PIK3CA, that have survival benefits. This meta-analysis included 17 recent clinical studies with large sample sizes to investigate the effects of aspirin on the long-term survival of esophageal, gastric and colorectal cancers. Although the studies included were retrospective studies, they were of high quality and had large sample sizes. The results indicated that postdiagnosis aspirin use may improve OS and CSS in patients with colorectal cancer but not in patients with esophageal cancer or gastric cancer. Subgroup analysis
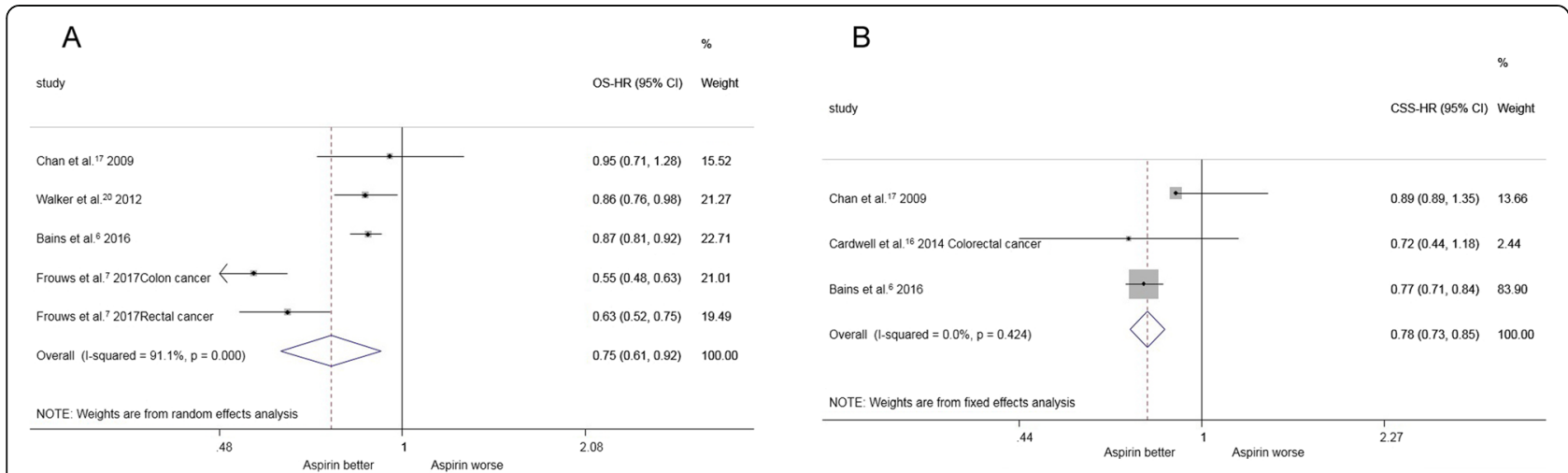

Fig. 4 a both pre and post-diagnosis aspirin use and overall survival for colorectal cancer. $\mathbf{b}$ both pre and post -diagnosis aspirin use and cancer specific survival for colorectal cancer 


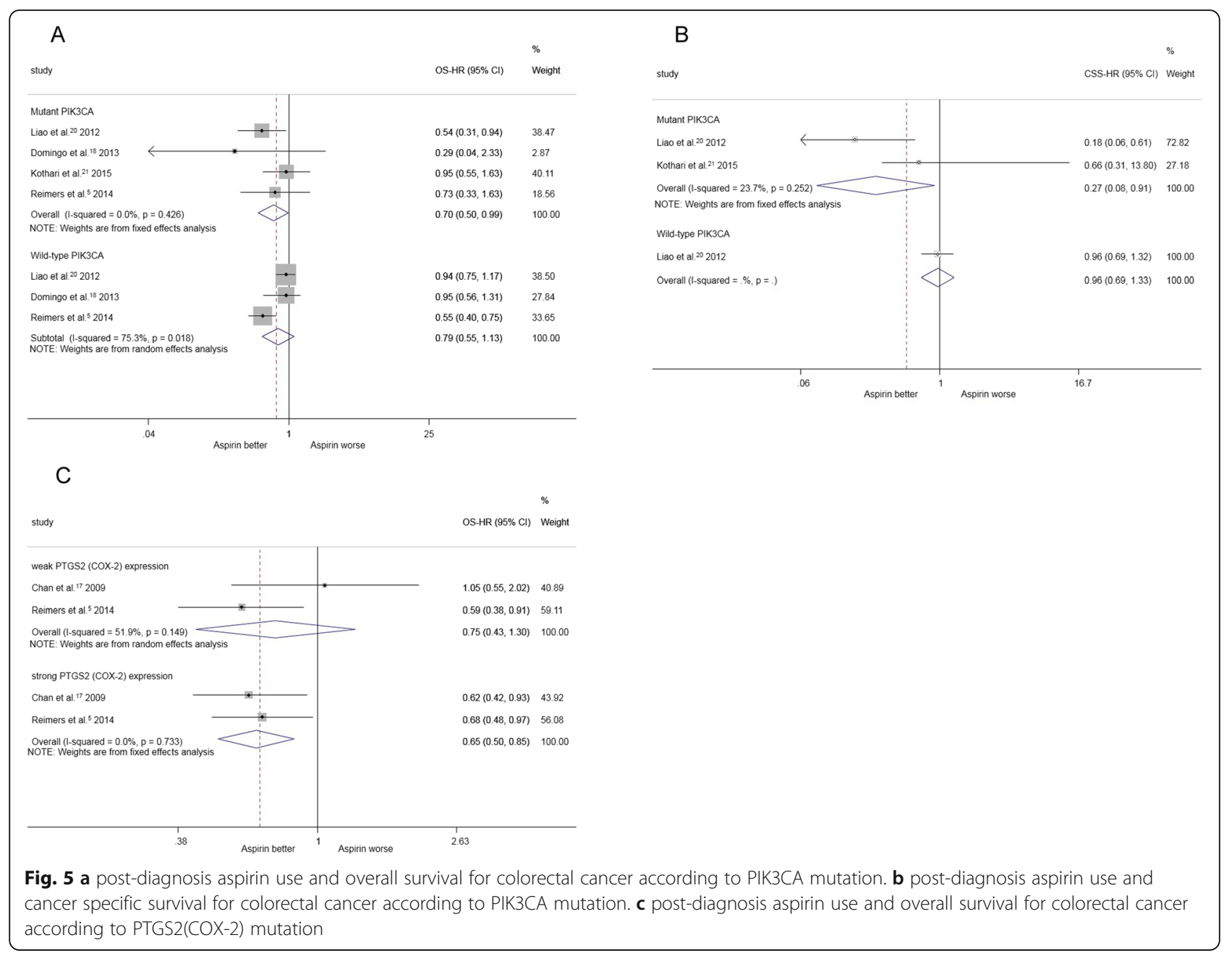

indicated that postdiagnosis aspirin use could prolong the long-term survival of patients with PIK3CA gene mutations and high expression of PTGS2 (COX-2).

A Dutch cohort study [7] that involved 946 patients with esophageal cancer and 750 patients with gastric cancer demonstrated that postdiagnosis aspirin use significantly reduced mortality in esophageal cancer $[\mathrm{HR}=$ 0.42 , 95\% CI $(0.30-0.57)]$ but failed to observe reduced mortality in gastric cancer $[\mathrm{HR}=0.87,95 \%$ CI 0.47 1.61]. Additionally, a British study [11] that included4654 patients with esophageal cancer and 3833 patients with gastric cancer observed that low-dose aspirin use did not reduce mortality in these patients. The present study also found that aspirin did not improve the overall survival rate of patients with esophageal and gastric cancer. Although the original studies had high quality and large sample sizes, more RCTs and evidencebased studies are needed because there are few studies that have focused on the long-term survival of patients with esophageal or gastric cancer.
Previous prospective studies [25, 26] have observed that aspirin can reduce colorectal adenomas and reduce the risk of colorectal adenomas recurrence. Most studies have found that aspirin should be used at least one year. The optimal dosage and duration is not consistent and large-scale prospective studies are still needed. This meta-analysis further supports that postdiagnosis aspirin use can improve the long-term survival of patients with colorectal cancer; however, prediagnosis aspirin use cannot improve the long-term survival of patients with colorectal cancer. As aspirin can lead to gastrointestinal bleeding and other side effects, it remains unclear whether low-dose aspirin can achieve adequate antitumor effects. Therefore, the long-term survival of patients with colorectal cancer needs to be evaluated with aspirin in the optimal dose and the best course of treatment. Moreover, side effects on the survival benefit of patients need to be investigated in the future. The daily dose of aspirin in the included observational studies was $75 \mathrm{mg}-$ $325 \mathrm{mg}$, and studies [27, 28] have shown that $81 \mathrm{mg}$ 


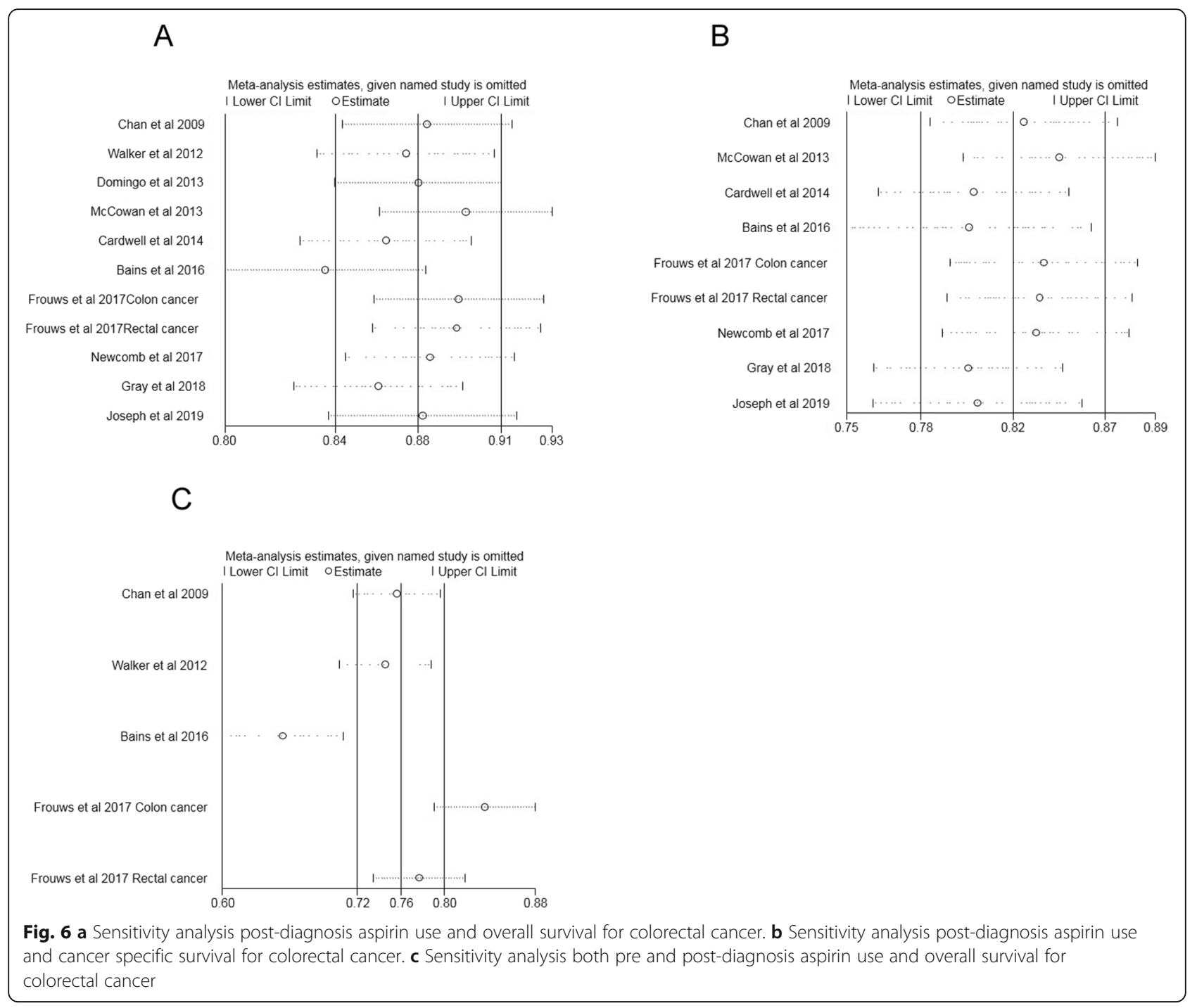

aspirin is sufficient to inhibit rectal mucosal PGE2 production. The US Preventive Services Working Group [29] recommends $81 \mathrm{mg}$ as a prescription dose for aspirin for the primary prevention of cardiovascular diseases and colorectal cancer. However, due to data limitations, a dose-response analysis between aspirin use and the long-term survival of patients with colorectal cancer was difficult to ascertain in the present study, and the optimal course of aspirin treatment needs to be investigated. We perform stratified analysis according to tumor stages. In patients with I-IV, aspirin may increase the overall survival $(\operatorname{HR}[0.88(0.79,0.98)])$ and cancerspecific survival (HR $[0.85(0.74,0.98)])$ as shown in supplement Figure 3 . We found that aspirin may increase CSS HR $[0.73(0.63,0.85)]$ in stage II patients, but there was no survival benefit in other stages. Due to the limited literatures and high heterogeneity, more literatures need to be included for further analysis. Because the inclusion studies did not provide detailed information, it was impossible to conduct a subgroup analysis based on whether surgery or chemotherapy.

The mechanism of action of aspirin in the treatment of colorectal cancer is unclear. Some biomarkers can be used to predict the survival benefit of aspirin in colorectal cancer, including PTGS2 (COX-2) expression and the effects of the PIK3CA gene. The anti-inflammatory effects of aspirin are mediated through direct inhibition of COX-1 and COX- 2 [30-32]. PTGS2 (COX-2) promotes the inflammatory response and cell proliferation, and high expression of PTGS2 (COX-2) is associated with poor survival in patients with colorectal cancer [33, 34]. The up-regulation of PI3K enhances PTGS2 (COX-2) activity and prostaglandin synthesis and plays an important role in the signal transduction pathway of tumorigenesis $[35,36]$. According to the subgroup analysis in our study, the effects of aspirin use on PIK3CA gene mutation and survival of patients with high expression of PTGS2 (COX-2) was different from that of patients with wildtype PIK3CA and 
PTGS2 (COX-2)-negative colorectal cancer. These findings provide a basis for the use of aspirin in patients with different types of mutations in colorectal cancer and the result can be used as a preliminary basis for further research.

Due to the bias of retrospective articles, it is necessary to perform randomised prospective studies to validate these data. At present, many clinical trials about aspirin and GI malignancies have not been completed. The ASAC trial (NCT03326791) are the first clinical interventional trial to assess the beneficial role of ASA in recurrence of CRC liver metastases and survival. Add-Aspirin (NCT02804815) aims to assess whether regular aspirin use after standard curative therapy can prevent recurrence and improve survival in individuals with non-metastatic common tumours. ASPIK French trial (NCT02945033) investigate Aspirin Versus Placebo in Resected Colon Cancer With PI3K Mutation Stage III or II High Risk. We also look forward to more prospective studies supporting the impact of aspirin on the prognosis of GI malignancies.

There were some limitations in this study. First, because the original studies were retrospective, there was some publication bias and selection bias. Second, due to the different definitions of aspirin use in the literature, the inclusion and exclusion criteria of the original studies were inconsistent; such differences may lead to deviations in the results. In addition, the number of studies involved was relatively small. Other potential confounding factors include the staging of tumors, whether surgery was performed, whether chemotherapy was performed, and the location of colorectal tumors. Because the included studies did not provide detailed information, it was impossible to conduct a subgroup analysis according to whether surgery, whether chemotherapy, the dosage, duration and reason for taking aspirin.

\section{Conclusion}

In conclusion, based on the results of this study, aspirin can improve OS and CSS in patients with colorectal cancer after diagnosis, especially in those with PIK3CA gene mutations and high PTGS2 (COX-2) gene expression, but it cannot improve OS in patients with esophageal cancer and gastric cancer. The results provide a theoretical basis for the conductance of future RCTs. If RCTs can further confirm that aspirin can improve the long-term survival of patients with colorectal cancer, such therapies will have important clinical significance and socioeconomic value for patients with colorectal cancer because aspirin is inexpensive.

\section{Supplementary information}

Supplementary information accompanies this paper at https://doi.org/10. 1186/s12885-020-07117-4.

Additional file 1: Figure 1A. Flow diagram of the selection process of gastric cancer. Figure 1B. Flow diagram of the selection process of esophageal cancer. Figure 1C. Flow diagram of the selection process of colorectal cancer.

Additional file 2: Supplementary file 2. Post-diagnosis aspirin use and overall survival for esophageal cancer according to pathologic type. A subgroup analysis was conducted according to the pathologic type of esophageal cancer. The estimated pooled HRs showed no significant differences were seen between the two groups $[\mathrm{HR}=1.05,95 \% \mathrm{CI}(0.92$, 1.20)]of esophageal adenocarcinoma. The estimated pooled HRs showed no significant differences were seen between the two groups $[\mathrm{HR}=0.89$, $95 \% \mathrm{Cl}(0.74,1.07)]$ of esophageal squamous cell carcinoma.

Additional file 3: Figure 3A aspirin use and overall survival for colorectal cancer according to tumor stage. Figure $\mathbf{3 B}$ aspirin use and cancer specific survival for colorectal cancer according to tumor stage.

\section{Abbreviations}

PPI: Proton Pump Inhibitor; RRs: Pooled risk ratios; OS: Overall survival; CSS: Cancer-specific survival; NOS: Newcastle-Ottawa Scale; RCTs: Random control trials; ASA: Acetylsalicylic acid; GI: Gastrointestinal;

NSAIDs: Nonsteroidal Antiinflammatory Drugs

\section{Acknowledgements}

The authors thank all the medical staff who contributed to the maintenance of the medical record database.

\section{Authors' contributions}

All authors have read and approved the manuscript. CMH, JLL and JXL conceptualized and designed the study, acquired and analysed data, interpreted the study results, drafted the manuscript and critically revised the manuscript for important intellectual content. $\mathrm{CHZ}$ and PL acquired and analysed data, interpreted the study results and critically revised the manuscript for important intellectual content. JWX and JBW designed the study, interpreted the study results and critically revised the manuscript for important intellectual content. JL and QYC designed the study, interpreted the study results and critically revised the manuscript for important intellectual content. LLC and ML conceptualized and designed the study, interpreted the study results and critically revised the manuscript for important intellectual content.

\section{Funding}

Scientific and technological innovation joint capital projects of Fujian Province, China (No.2016Y9031). Construction Project of Fujian Province Minimally Invasive Medical Center (No. [2017]171). Fujian province medical innovation project (2015-CXB-16). The funding bodies were neither involved in the design of the study, nor in the collection, analysis, or interpretation of data nor in the writing of the manuscript.

\section{Availability of data and materials}

The data that support the findings of this study are available from the corresponding author upon reasonable request.

Ethics approval and consent to participate

Not applicable.

Consent for publication

Not applicable.

\section{Competing interests}

The authors have no conflicts of interest associated with the publication of this manuscript to declare. The authors report no relevant financial disclosures related to this current work.

\section{Author details}

'Department of Gastric Surgery, Fujian Medical University Union Hospital, No.29 Xinquan Road, Fuzhou 350001, Fujian Province, China. ${ }^{2}$ Department of General Surgery, Fujian Medical University Union Hospital, Fuzhou, Fujian Province, China. ${ }^{3}$ Key Laboratory of Ministry of Education of Gastrointestinal Cancer, Fujian Medical University, Fuzhou, Fujian Province, China. 
Received: 28 July 2019 Accepted: 26 June 2020

Published online: 09 July 2020

\section{References}

1. Bray F, Ferlay J, Soerjomataram I, et al. Global cancer statistics 2018: GLOBOCAN estimates of incidence and mortality worldwide for 36 cancers in 185 countries. CA Cancer J Clin. 2018;68(6):394-424. https://doi.org/10. 3322/caac.21492.

2. Algra AM, Rothwell PM. Effects of regular aspirin on long-term cancer incidence and metastasis: a systematic comparison of evidence from observational studies versus randomised trials. Lancet Oncol. 2012;13(5): 518-27. https://doi.org/10.1016/S1470-2045(12)70112-2.

3. Li P, Wu H, Zhang $H$, et al. Aspirin use after diagnosis but not prediagnosis improves established colorectal cancer survival: a meta-analysis. Gut. 2015; 64(9):1419-25. https://doi.org/10.1136/gutjnl-2014-308260.

4. Din FV, Theodoratou E, Farrington SM, et al. Effect of aspirin and NSAIDs on risk and survival from colorectal cancer. Gut. 2010;59(12):1670-9. https://doi. org/10.1136/gut.2009.203000.

5. Reimers MS, Bastiaannet E, Langley RE, et al. Expression of HLA class I antigen, aspirin use, and survival after a diagnosis of colon cancer. JAMA Intern Med. 2014;174(5):732-9. https://doi.org/10.1001/jamainternmed.2014. 511.

6. Bains SJ, Mahic M, Myklebust TA, et al. Aspirin as secondary prevention in patients with colorectal Cancer: an unselected population-based study. J Clin Oncol. 2016;34(21):2501-8. https://doi.org/10.1200/JCO.2015.65.3519.

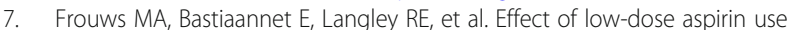
on survival of patients with gastrointestinal malignancies; an observational study. Br J Cancer. 2017;116(3):405-13. https://doi.org/10.1038/bjc.2016.425.

8. Moher D, Liberati A, Tetzlaff J, et al. Preferred reporting items for systematic reviews and meta-analyses: the PRISMA statement. PLoS Med. 2009;6(7): e1000097. https://doi.org/10.1371/journal.pmed.1000097.

9. Stang A. Critical evaluation of the Newcastle-Ottawa scale for the assessment of the quality of nonrandomized studies in meta-analyses. Eur J Epidemiol. 2010;25(9):603-5. https://doi.org/10.1007/s10654-010-9491-z.

10. Higgins JP, Thompson SG. Quantifying heterogeneity in a meta-analysis. Stat Med. 2002;21(11):1539-58. https://doi.org/10.1002/sim.1186.

11. Spence AD, Busby J, Johnston BT, et al. Low-dose aspirin use does not increase survival in 2 independent population-based cohorts of patients with esophageal or gastric Cancer. Gastroenterology. 2018;154(4):849-60 e1. https://doi.org/10.1053/j.gastro.2017.10.044.

12. Macfarlane TV, Murchie P, Watson MC. Aspirin and other non-steroidal antiinflammatory drug prescriptions and survival after the diagnosis of head and neck and oesophageal cancer. Cancer Epidemiol. 2015;39(6):1015-22. https://doi.org/10.1016/j.canep.2015.10.030.

13. Coghill AE, Newcomb PA, Campbell PT, et al. Prediagnostic non-steroidal anti-inflammatory drug use and survival after diagnosis of colorectal cancer. Gut. 2011;60(4):491-8. https://doi.org/10.1136/gut.2010.221143.

14. Zell JA, Ziogas A, Bernstein $L$, et al. Nonsteroidal anti-inflammatory drugs: effects on mortality after colorectal cancer diagnosis. Cancer. 2009;115(24): 5662-71. https://doi.org/10.1002/cncr.24705.

15. Cardwell CR, Kunzmann AT, Cantwell MM, et al. Low-dose aspirin use after diagnosis of colorectal cancer does not increase survival: a case-control analysis of a population-based cohort. Gastroenterology. 2014;146(3):700-8 e2. https://doi.org/10.1053/j.gastro.2013.11.005

16. Chan AT, Ogino S, Fuchs CS. Aspirin use and survival after diagnosis of colorectal cancer. JAMA. 2009:302(6):649-58. https://doi.org/10.1001/jama. 2009.1112.

17. Domingo E, Church DN, Sieber O, et al. Evaluation of PIK3CA mutation as a predictor of benefit from nonsteroidal anti-inflammatory drug therapy in colorectal cancer. J Clin Oncol. 2013;31(34):4297-305. https://doi.org/10. 1200/JCO.2013.50.0322.

18. McCowan C, Munro AJ, Donnan PT, et al. Use of aspirin post-diagnosis in a cohort of patients with colorectal cancer and its association with all-cause and colorectal cancer specific mortality. Eur J Cancer. 2013;49(5):1049-57. https://doi.org/10.1016/j.ejca.2012.10.024.

19. Walker AJ, Grainge MJ, Card TR. Aspirin and other non-steroidal antiinflammatory drug use and colorectal cancer survival: a cohort study. $\mathrm{Br}\rfloor$ Cancer. 2012;107(9):1602-7. https://doi.org/10.1038/bjc.2012.427.

20. Kothari $\mathrm{N}$, Kim $\mathrm{R}$, Jorissen $\mathrm{RN}$, et al. Impact of regular aspirin use on overall and cancer-specific survival in patients with colorectal cancer harboring a
PIK3CA mutation. Acta Oncol. 2015;54(4):487-92. https://doi.org/10.3109/ 0284186X.2014.990158.

21. Liao $X$, Lochhead $P$, Nishihara $R$, et al. Aspirin use, tumor PIK3CA mutation, and colorectal-cancer survival. N Engl J Med. 2012;367(17):1596-606. https:// doi.org/10.1056/NEJMoa1207756.

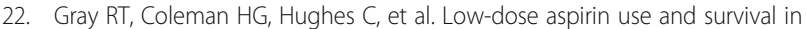
colorectal cancer: results from a population-based cohort study. BMC Cancer. 2018;18(1):228. https://doi.org/10.1186/s12885-018-4142-y.

23. Hua X, Phipps Al, Burnett-Hartman AN, et al. Timing of aspirin and other nonsteroidal anti-inflammatory drug use among patients with colorectal Cancer in relation to tumor markers and survival. J Clin Oncol. 2017;35(24): 2806-13. https://doi.org/10.1200/JCO.2017.72.3569.

24. Sung JJY, Ho JMW, Chan FCH, et al. Low-dose aspirin can reduce colorectal cancer mortality after surgery: a 10-year follow-up of 13528 colorectal cancer patients. J Gastroenterol Hepatol. 2019;34(6):1027-34. https://doi.org/ 10.1111/jgh.14562.

25. Sandler RS, Halabi S, Baron JA, et al. A randomized trial of aspirin to prevent colorectal adenomas in patients with previous colorectal cancer. N Engl J Med. 2003;348(10):883-90. https://doi.org/10.1056/NEJMoa021633.

26. Baron JA, Cole BF, Sandler RS, et al. A randomized trial of aspirin to prevent colorectal adenomas. N Engl J Med. 2003;348(10):891-9. https://doi.org/10. 1056/NEJMoa021735.

27. Ruffin MT, Krishnan $\mathrm{K}$, Rock $\mathrm{CL}$, et al. Suppression of human colorectal mucosal prostaglandins: determining the lowest effective aspirin dose. J Natl Cancer Inst. 1997;89(15):1152-60.

28. Sample D, Wargovich M, Fischer SM, et al. A dose-finding study of aspirin for chemoprevention utilizing rectal mucosal prostaglandin E(2) levels as a biomarker. Cancer Epidemiol Biomarkers Prev. 2002;11(3):275-9.

29. Bibbins-Domingo K. Force USPST. Aspirin use for the primary prevention of cardiovascular disease and colorectal Cancer: U.S. preventive services task force recommendation statement. Ann Intern Med. 2016;164(12):836-45. https://doi.org/10.7326/M16-0577.

30. Chen WS, Wei SJ, Liu JM, et al. Tumor invasiveness and liver metastasis of colon cancer cells correlated with cyclooxygenase-2 (COX-2) expression and inhibited by a COX-2-selective inhibitor, etodolac. Int J Cancer. 2001;91(6): 894-9.

31. Brown JR, DuBois RN. COX-2: a molecular target for colorectal cancer prevention. J Clin Oncol. 2005;23(12):2840-55. https://doi.org/10.1200/JCO. 2005.09.051.

32. Wang $\mathrm{D}$, Dubois RN. The role of COX-2 in intestinal inflammation and colorectal cancer. Oncogene. 2010;29(6):781-8. https://doi.org/10.1038/onc. 2009.421.

33. Dong M, Johnson M, Rezaie A, et al. Cytoplasmic phospholipase A2 levels correlate with apoptosis in human colon tumorigenesis. Clin Cancer Res. 2005;11(6):2265-71. https://doi.org/10.1158/1078-0432.CCR-04-1079.

34. Soumaoro LT, Uetake H, Higuchi T, et al. Cyclooxygenase-2 expression: a significant prognostic indicator for patients with colorectal cancer. Clin Cancer Res. 2004;10(24):8465-71. https://doi.org/10.1158/1078-0432.CCR-040653.

35. Samuels $Y$, Wang $Z$, Bardelli $A$, et al. High frequency of mutations of the PIK3CA gene in human cancers. Science. 2004;304(5670):554. https://doi.org/ 10.1126/science.1096502

36. Kaur J, Sanyal SN. PI3-kinase/Wnt association mediates COX-2/PGE(2) pathway to inhibit apoptosis in early stages of colon carcinogenesis: chemoprevention by diclofenac. Tumour Biol. 2010;31(6):623-31. https://doi. org/10.1007/s13277-010-0078-9.

\section{Publisher's Note}

Springer Nature remains neutral with regard to jurisdictional claims in published maps and institutional affiliations. 